\title{
Der Begriff der Moderne \\ anhand von Alfred Anderschs Roman \\ „Sansibar oder der letzte Grund"
}

vorgelegt von

\section{Hend Ismaiel Zaki Mohammed Mohran}

\section{Zur Textgattung des Romans}

Der Roman ist eine Großform erzählender Prosa und gehört zur Grundgattung der Epik. Das Wort stammt aus dem Altfranzösischen ,romance“ und bedeutet „Erzählung in Versen oder Prosa“. Im Mittelalter began man in Europa, zwischen dem Volk und den Intellektuellen zu unterscheiden. Man nannte die Sprache des Volkes lingua romana, und die Sprache der Intellektuellen Latein. Das Wort Roman kommt also von lingua romana, weil der Roman eine Literaturform für das lesende Volk und nicht für die Intellektuellen war. Andere Formen der Literatur wie Lyrik und Epik wurden hauptsächlich für Intellektuelle geschrieben und von ihnen gelesen, auch wenn sie nicht auf Latein waren. ${ }^{1}$

Ab dem 12. Jahrhundert bezeichnete romance die in romanischer Volkssprache verfassten Schriften in Versen oder Prosa. Als Prosaform hat der Roman in der Neuzeit gemeinsam mit der Novelle und der Erzählung das Epos abgelöst. Nach einem allgemeinen Verständnis ist jedes längere Werk erzählender Prosa ein Roman. $^{2}$

Im 17. Jahrhundert erschien der Begriff „Roman“ in Deutschland. 1668 wird der erste deutschsprachige Roman, „Der abenteuerliche Simplicissimus“ von Hans Jakob Christoffel von Grimmelshausen veröffentlicht. Er beschreibt, was ein naiver junger Mann in den Wirren und Grausamkeiten des 30 jährigen Krieges erlebt. ${ }^{3}$

Im Laufe des 18. Jahrhunderts wird der Roman als Medium der Unterhaltung und Belehrung und schließlich auch als Kunstform erkannt. Um 1740 erschienen wenige Romane in Deutschland, die kaum eine Bedeutung hatten, doch in den folgenden Jahren nahm seine Bedeutung immer mehr zu. ${ }^{4}$

Allerdings schrieben viele Romanschriftsteller des 20. Jahrhunderts eine neue Art des Romans und versuchen nicht mehr, diese totale Welt zu zeigen, oder eine ganze Welt zu schaffen, weil sie meinen, der Mensch kann das in der modernen Welt nicht mehr verstehen. Oft betonen diese Autoren die Innenwelt der Charaktere. ${ }^{5}$ Wolfgang Kayser definiert den Roman als die 
von einem fiktiven, persönlichen Erzähler vorgetragene, einen persönlichen Leser einbeziehende Erzählung von Welt, soweit sie als persönliche Erfahrung fassbar wird. Der einzelne Roman gewinnt Geschlossenheit dadurch, dass er entweder eine Handlung oder den Raum (d.h. die Vielzahl von Räumlichkeiten) oder eine Figur zur strukturtragenden Schicht macht. ${ }^{6}$

Der Roman ist eine ausführliche Geschichte, die das abenteuerliche Leben eines einzelnen oder mehrere Menschen beschreibt und zeigt wie sich die Romanfiguren in den unterschiedlichen Lebenssituationen verhalten, daher umfasst er keine Einzelergebnisse, sondern Zusammenhänge.

Der Roman zeigt ein breites Totalbild der Welt, der Zeit und der Gesellschaft in bunter Handlungsfülle, ohne geschlossenen Geschehensaufbau. Er zeigt meistens innere und äußere Welten. Der Erzähler beschreibt nicht nur das, was den Charakteren geschieht, sondern auch das, was die Charaktere denken und fühlen.

Der Roman ist die bekannteste und die Literatur dominierende epische Gattung. Als epische Großform unterscheidet er sich von den Kleinformen (Novelle, Erzählung, Märchen, Kurzgeschichte, Sage, Legende, Fabel, Anekdote und Witz) zunächst durch seinen äußeren Umfang; dass der Umfang eine relative Größe und daher nicht genau bestimmbar ist, ist unbestritten. Der äußere Umfang verweist auf einen inneren: auf die Vielfalt und Fülle der Themen und Handlungen verbunden mit einer Vielfalt von Schreibweisen und einer vielschichtigen Gestaltung und Struktur. Da er in Prosa geschrieben ist und nicht in Versen, unterscheidet er sich von der anderen epischen Großform, dem Epos oder Versroman. ${ }^{7}$

Der Roman hat typische Merkmale gegenüber anderem prosaischen Gattungen. Im Gegensatz zum Epos tritt im Roman verstärkt eine individuell gestaltete Persönlichkeit einer als problematisch empfundenen Welt entgegen. Der Konflikt zwischen Individuum und Gesellschaft ist häufig ein zentrales Motiv und wird mit wechselnden Außen- und Innenperspektiven verdeutlicht. Im Unterschied zur Novelle gibt es im Roman meist kein herausgehobenes Ereignis in Form eines dramatischen Höhe- oder Wendepunktes. Stattdessen bildet ein umfassender Ausschnitt aus dem Leben einer Person mit ihrem sozialen Umfeld die Grundlage. ${ }^{8}$

Die Handlung des Romans kann in einer geraden Linie verfolgen oder auch in der Zukunft vorwegnehmen oder bereits Vergangenes hervorbringen. Der Erzähler kann im Roman verborgen sein oder die Handlung kommentieren. Der Roman spiegelt viele Kulturen durch seine Handlungen und seine Schreibweisen wider. Heutzutage sind Romane die meist gelesene Literatur und behaupten sich weiterhin erfolgreich gegen populäre Erzählmedien wie das Kino oder das Fernsehen. ${ }^{9}$ 
Der Roman ist die offenste Form der Darstellung, er kann vom Bericht über die Autobiographie bis zum dramatischen Dialog reichen, er vermag Elemente der Lyrik und Tragik einzunehmen. ${ }^{10}$

Romane lassen sich inhaltlich oder formal in verschiedene Kategorien einteilen. Es gibt verschiedene Wirkungsabsichten im Roman z.B. lehrreiche, unterhaltende, satirische, idealistische, empfindsame, realistische usw. Nach dem Inhalt unterscheidet man unter anderem zwischen den Genres: Abenteuerroman, Ritterroman, Kriegsroman, Kriminalroman, Liebesroman, Familienroman, Reiseroman, historischer Roman, philosophischer Roman usw. Nach der formalen Gestaltung lassen sich Romane zum Beispiel in Bezug auf ihre äußere Erzählform (Briefroman, Tagebuchroman), und hinsichtlich der Erzählperspektive (IchErzähler, allwissender auktorialer Erzähler, personale Erzählsituation) einteilen. ${ }^{11}$

\section{Der Roman und die Moderne}

Wenn man von einem modernen Roman spricht, so möchte man meinen, es handele sich um einen Roman, der in der Epoche der Moderne verfasst wurde. Als Beginn der Moderne in der Romanliteratur lassen sich recht präzise die Jahre 1910 bis 1912 angeben, in denen die ersten Werke mit typisch modernen Eigenschaften veröffentlicht wurden. Zu den ersten Romanen dieser Art zählt Rainer Maria Rilkes „Die Aufzeichnungen des Malte Laurids Brigge“ und Carl Einsteins „Bebuquin oder die Dilettanten des Wunders“, in denen bereits einige der Stilmittel und Methoden eingesetzt wurden. Die von Rilke und Einstein genutzten Mittel stammen jedoch schon aus der Romantik, in der bereits einige Autoren vom Einsatz dieser Techniken kündeten, diese jedoch nie konsequent anwendeten. Man kann also durchaus behaupten, die Idee vom modernen Roman sei bereits in der Zeit der Romantik geboren worden, jedoch erst im 20. Jahrhundert durchsetzbar gewesen. Alle diese Romane vereinen mehr oder weniger die Eigenschaften, die der Moderne zugerechnet werden können. Eine Großzahl der verfassten Bücher hat als zentrales Thema den Zerfall der Kultur oder der Gesellschaft. ${ }^{12}$

Wesentlich sind zwei Tendenzen, die für die Romangestaltung im 20. Jahrhundert bestimmend sind. Erstens: Die Erringung einer nahezu uneingeschränkten Freiheit für den Erzähler, für die Gestaltung des Helden, des Geschehens, der Komposition des Romans und für die Hereinnahme der unterschiedlichsten Darstellungsmittel, Stilelemente und Sprechformen. Und zweitens: Die komplexe Wahrheit über Mensch und Welt wird unmittelbar dargestellt. Das geschieht notfalls unter Verzicht auf äußere Realitätstreue, im Extrem in allen Einzelaspekten des Romans. ${ }^{13}$

Der Autor schildert die Welt aus der Perspektive der Figur. Dem Leser wird immer wieder zugemutet, sich um ein subtiles Verständnisvon Aussage und Kompositionsform zu bemühen. Das bedeutet für das Helden- und Menschenbild des modernen Romans: Der Einzelne ist weder als individueller Charakter noch als Typus, sondern vielmehr in seiner menschlichen Substanz interessant, die 
wesentlich mehr von seiner Beziehung zur Gesellschaft oder Außenwelt überhaupt abhängig erscheint als von Familie und Tradition. Der Einzelne wird stärker von seinem Innenleben, von Bewusstsein und Lebensgefühl her gesehen als von möglichen Aktivitäten.

Die Frage nach dem Menschen als mehr oder weniger genau bestimmbarem Wesen ist eines der zentralen Probleme des modernen Romans. Das gilt sowohl inhaltlich wie formal, denn die Struktur des Ganzen ist entscheidend von der Konzeption dieses im Mittelpunkt stehenden Dichtungsgegenstandes abhängig. Die Gestaltung der Heldenfigur dient in zunehmendem Maße der Frage nach den Möglichkeiten und Grenzen des Menschen in der gegenwärtigen Zeitsituation. Demgemäß werden sein Selbstverständnis und sein Lebensgefühl wichtiger als singuläre Erlebnisse von geringer Repräsentanz. ${ }^{14}$

Wichtigster Grundsatz für die Gestaltung eines modernen Romans ist die Freiheit des Autors in einem Stück die verschiedensten Gestaltungsformen, was den Erzähler die Hauptfigur oder die Nutzung der verschiedene Stilmittel angeht, zu nutzen. Hinzu kommt, dass der moderne Erzähler nicht mehr der Herr seiner Romanwelt ist, sondern verbogen und verändert wird, wie es gerade nötig ist, nur um der vom Autor gewählten Thematik zu dienen.

Um das Innenleben eines Charakters ausführlich genug zu beschreiben reduziert der moderne Romanautor die tatsächliche Realität auf ein Minimum und konzentriert sich auf die Erfahrung aus der Realität, sei diese nun realistisch oder nicht. Es geht dem Autor des modernen Romans also primär um die innere Situation des einzelnen Menschen und seiner Beziehung zur Gesellschaft und nicht wie im klassischen Roman um die Beziehung zur Familie oder Tradition. Im modernen Roman findet deshalb eine Abwendung von der Betrachtung der Taten und Handlungen der Hauptperson und eine Zuwendung zur Betrachtung des Bewusstsein und des Lebensgefühls statt. Im modernen Roman scheint die Beschreibung eines Zustandes oder eines Problems durch eine bestimmte Situation wesentlich an Bedeutung und verdrängt somit den chronologischen Ablauf, der bisher für den Ablauf des Geschehens wichtig war. ${ }^{15}$

Der Held traditioneller Romane erlebt in seiner gewohnten Umgebung ein spezielles Schicksal. Der „neue“ Held des modernen Romans lebt meistens in einer ihm nicht vertrauten Umwelt, in der er meist mehr oder weniger herumirrt und ist zumindest teilweise nur noch Repräsentant des allgemeinen Menschen. Eine andere neue Heldenfigur ist der Antiheld, der gegen sein Schicksal ankämpft.

$\mathrm{Zu}$ den bevorzugten Themen des modernen Romans ist zu sagen, dass grundsätzlich der gedankliche Innenraum des Menschen und sein Bewusstsein bezüglich der Welt oder seinem Ich die größte Rolle spielt. Eine Besonderheit des deutschen Romans ist die verstärkte Aufnahme der gesellschaftlichen Verhältnisse als Themenbereich. 


\section{Merkmale des modernen Roman im Roman „Sansibar oder der letzte Grund"}

Alfred Anderschs 1957 veröffentlichtes Werk „Sansibar oder der letzte Grund“ handelt von fünf, in ihren Absichten sehr unterschiedlichen Menschen, die sich 1937 in dem kleinen Ostseestädtchen Rerik aufhalten. Unter dem Eindruck des Nationalsozialismus begegnet dem Leser der Schiffsjunge, der aus Langeweile aus Rerik weg will, der Kommunist Gregor, der sich von seiner Partei abzuwenden beginnt, der Pfarrer Helander, der im ersten Weltkrieg sein Bein verloren hat, der Fischer Knudsen, den Gregor im Auftrag der Partei treffen will, und Knudsen geistig beeinträchtigte Frau Bertha. Schließlich befindet sich noch die wohlhabende Jüdin Judith in Rerik, die eine Flucht nach Schweden beabsichtigt. Als letztes ist da noch eine Holzfigur mit dem Namen „lesender Klosterschüler“, die bedroht ist, von den Nationalsozialisten geholt zu werden.

Der Roman ,Sansibar oder der letzte Grund “ trägt viele Züge modernen Erzählens, weist aber auch traditionelle Elemente auf. $\mathrm{Zu}$ den modernen Aspekten zählen verschiedene Herangehensweise: ${ }^{16}$

Die personale Erzählsituation ist dadurch gekennzeichnet, dass das Geschehen fast nur aus der Sicht einer beteiligten Person geschildert wird. Der Leser erhält die Illusion, er befände sich selbst auf dem Schauplatz des Geschehens. Nicht die berichtende, sondern die szenische Darstellung ist die dem personalen Roman entscheidende Grundform. ${ }^{17}$

Der personale Erzähler ist ein Person, die in die Handlung integriert ist alles aus ihrer Sicht subjektiv erzählt. Bei der personalen Erzählform wird die Handlung aus der Sicht einer bestimmten Person, die in der Handlung eine Rolle spielt, erzählt. Im Gegensatz zum auktorialen Erzähler ist die Person also nicht allwissend, sondern weiß logischerweise nur das, was sie selbst erlebt.

Der personale Erzähler schlüpft in eine oder mehrere Personen und erzählt die Geschichte aus deren Perspektive, aber nicht in der grammatischen Ich-Form, sondern in der 3. Person ("er", "sie"). Das Spannende an dieser Erzählperspektive ist, dass der Erzähler nur mit den Augen der gewählten Figur in die Welt blickt.

Dieser Erzähler vermittelt dadurch dem Leser wesentlich mehr Emotionen bzw. lässt diesen viel stärker an den Ereignissen inklusive Überraschungen und Wendepunkten teilhaben. Er ist ebenfalls subjektiv, dass heißt der Leser erfährt das Geschehen aus der Sicht der Person, die ebenfalls alles nach der eigenen Meinung bewerten und beurteilen kann ("Subjektives Ich").

Der Roman „Sansibar oder der letzte Grund“ wird aus der Perspektive der fünf Hauptgestalten erzählt. Es handelt sich also um eine personale Perspektive. Der Roman beschränkt sich auf das, was sich im Inneren der Figuren spiegelt. Der Leser 
erfährt ausschließlich deren Wahrnehmungen, Gedanken und Handlungen. Es werden nicht fertige Ergebnisse eines Denkvorgangs präsentiert, es handelt sich viel mehr um die Wiedergabe des Denkvorgangs. ${ }^{18}$

Im Roman „Sansibar" tritt kein besonderer Erzähler auf, weder ein allwissender, auktorialer noch ein Ich-Erzähler. Der Erzähler tritt so weit hinter die Charaktere des Romans zurück, dass seine Anwesenheit dem Leser nicht mehr bewusst wird, dann eröffnet sich dem Leser die Illusion, er befände sich selbst auf dem Schauplatz des Geschehens oder er betrachte die dargestellte Welt mit den Augen einer Romanfigur, die jedoch nicht erzählt, sondern in deren Bewusstsein sich das Geschehen gleichsam abspielt. ${ }^{19}$

Der Leser soll die Weltsicht der Figuren genau nachvollziehen können. Er wird plötzlich und ohne Einführung in die Gedankenwelt der Figuren hineinversetzt und muss sich selbst zurechtfinden.

Das Romangeschehen spiegelt sich nicht nur in einer Romanfigur, sondern im Bewusstsein aller fünf Hauptpersonen wider. Was der Autor als Romanhandlung vermitteln will, sehen wir immer wieder abwechselnd aus der Perspektive des Junges, Gregors, Helander, Judiths und Knudsens. Diese Personen spiegeln nicht nur die Handlung wider, sondern reflektieren, kommentieren, und bewerten sie auch. „Dann sah sie zum Fenster hinaus: ein Hohlziegeldach unter einem nördlichen, hellen, vollständig leeren Herbsthimmel “. ${ }^{20}$

Es gibt auch Passagen, in denen der Autor Situationen und Personen direkt darstellt:

Sie saß auf dem Bett eines Fremdenzimmers im >Wappen von Wismar< und kramte in ihrer Handtasche. Der Koffer stand neben der Tür, so, wie der Hausknecht ihn hingestellt hatte. (Sansibar, S. 17)

Andersch hat die einzelnen, meist recht kurzen Kapitel seines Romans mit dem Namen desjenigen überschrieben, aus dessen Blickwinkel das Geschehen gesehen wird, um dem Leser bei der mehrfachen Spiegelung des Romangeschehens die Orientierung zu erleichtern. Es ist eine der auffälligsten Eigenheiten dieses Romans, dass seine Kapitelüberschriften kein Angaben über den Inhalt, sondern ausschließlich über die erzählerische Perspektive machen.

Es ist hier wichtig, den Begriff Bewusstseinsstrom zu erwähnen, weil er ein wichtiges Merkmal im Roman ist. Damit wird gemeint, die möglichst realitätsnahe Abbildung der Gedankenwelt einer Figur, daher auch Brüche, Gedankensprünge, Assoziationsketten, unvermittelt einsetzende Erinnerungen und Rückblenden, sowie kreisende Wiederholungen. Bewusstseinsstrom ist eine Erzähltechnik, die in ungeordneter Folge Bewusstseinsinhalte einer oder mehrerer Figuren wiedergibt. 
Die Erzähltechnik des Bewusstseinsstroms ist die direkte Personenrede mit syntaktischer Unabhängigkeit, Verwendung des Präsens als Normaltempus und des Indikativ als Normalmodus. Zur Bezeichnung der denkenden Figur dient die erste Person, es wird also aus der Ich-Perspektive erzählt.

Der allgemeine Begriff für die Mitteilung unausgesprochener Gedanken der Figuren in Form von direkter Rede ist dabei ,innerer Monolog“. Der Begriff „Bewusstseinsstrom“ wird jedoch sehr uneinheitlich gebraucht und teilweise als Synonym für den inneren Monolog verwendet.

Die einzelnen Personen werden häufig im Bewusstseinsstrom anderer Personen gezeigt und ihre Erscheinung dabei zugleich kommentiert und bewertet:

Aha, dachte Knudsen, offenbar war die Stunde gekommen, in der dem stolzen Pfarrer Helander sein Verdun-Bein nichts mehr nützte. (Sansibar, S. 27)

Eine Jüdin, dachte Gregor, das ist ja eine Jüdin. Was will die hier in Rerik? Er sah Judith unter den Menschen stehen, die zusahen, wie der schwedische Dampfer anlegte. (Sansibar, S. 59)

Die Wahrnehmungen der einzelnen Hauptpersonen sind auch nur Auslöser von Gedankenassoziationen und Erinnerungen, die die entsprechende Person weit von der eigentlichen Romanhandlung fortführt. Ein Beispiel hierfür bietet sich am Beginn des Romans, als Gregor die Stadt Rerik sieht.

Es kam so, wie Gregor es sich vorgestellt hatte: die Kiefern hörten auf einmal auf, die Straße hob sich noch einmal auf den Rücken der Moräne, und von oben bot sich das erwartete Bild: die Weiden, die Koppeln, von schwarz- weißen Kühen und von Pferden gefleckt, dann die Stadt, dahinter das Meer, eine blaue Wand. (Sansibar, S. 21)

Rerik wird aus der Perspektive Gregors gezeigt. Für ihn ist Rerik nicht irgendeine Ortschaft, ihn faszinieren die Türme dieser Stadt auf besondere Weise. Er glaubt, dass solche Türme das Denken und Leben der Menschen beeinflussen, die unter ihnen wohnen. Er weiß auch, dass seine Auftraggeber im Zentralkomitee nicht so empfinden. Für sie ist der Ort Rerik nur ein Punkt im Netz ihrer Strategie. Rerik und die Türme sind ein ästhetisches und psychologisches Erlebnis.

Hier erscheint auch die Abwechslung von erlebter Rede und innerem Monolog. Die erlebte Rede gibt eher tiefere Denkprozesse wieder, der innere Monolog die daraus resultierenden Erkentnisse:

Nummer drei, dachte Gregor, während er das Mädchen beobachtete. Nummer drei, die fliehen will. Erst war es nur ich, dann ist der 
Klosterschüler dazugekommen, jetzt diese da. Ein reizender Land- man steht vor fremden Schiffen an, um es zu verlassen. Er blickte über den belebten Platz: nein, diese Leute alle wollten nicht fort, viele von ihnen mochten unzufrieden sein, aber sie kamen nicht auf die Idee, fortgehen zu wollen[...]. (Sansibar, S. 61)

Die perspektivische Darstellung, die die Welt immer wieder nur als Bild eines Individuums, als Spiegelung eines Bewusstseins zeigt, entspricht dem schon beschriebenen Eindruck von der Isoliertheit der einzelnen Personen dieses Romans. Jeder bewegt sich für sich allein im Raum, jeder sieht und hat nur seine eigene Welt und wird trotz aller Wahrnehmungen des Äußeren immer wieder zurückgeworfen auf sein eigenes Ich. Diese Isolation kann durchbrochen werden, wo die Kapitelüberschriften zwei oder mehrere Personen nennen.

Die Simultantechnik ist eine literarische Technik zur Erfassung der Gleichzeitigkeit verschiedener Ereignisse, die sich räumlich getrennt abspielen. Sie ist ein ästhetisches Verfahren, bei dem zeitlich, örtlich oder logisch auseinanderliegende Wirklichkeitsausschnitte oder Handlungs- und Gedankenfolgen zusammengerückt werden. Manchmal wird dasselbe Geschehen aus unterschiedlicher Perspektive geschildert, auch zur Verdeutlichung der Isolation der Personen. ${ }^{21}$

Helander ist auf dem Weg zu Knudsen. Die Beschreibung aus der Sicht Knudsens

Knudsen beobachtete ihn aus den Augenwinkeln, das konnte er sehen. Helander näherte sich ihm langsam, auf seinen Stock gestützt [...] Endlich stand Helander an der Kaimauer, dort, wo die Pauline festgemacht hatte. (Sansibar, S. 25)

Aus der Sicht Judiths wird das Geschehen so beschrieben,

Sie sah wieder zum Fenster hinaus. Der Geistliche, der mit dem Fischer gesprochen hatte, kam jetzt über den Platz. Er ging an einem Stock. Judith sah, daß er Schmerzen haben mußte, denn in seiner Haltung war etwas Angestrengtes, so, als müsse er sich beherrschen, um sich nicht völlig über seinen Stock zu krümmen. (Sansibar, S. 35)

Der Begriff Montage (frz., zu monter: „hinaufbringen, aufstellen“) ${ }^{22}$ bedeutet im allgemeinen Sinne „das Zusammenfügen von Einzelteilen zu einem Ganzen“, bzw. das „Zusammenbauen vorgefertigter Teile“. ${ }^{23}$ Sinngemäß sieht Volker Klotz die Montage als „die Tätigkeit, vorgefertigte Teile zu einem Ganzen zusammenzusetzen“. ${ }^{24}$

Die Montagetechnik ist eine Bezeichnung für das Zusammenfügen unterschiedlicher Texte oder Textteile, die unterschiedliche Inhalte beziehungsweise 
oft unterschiedliche Sprachebenen und -stile transportieren. Sie ist die Art und Weise, wie etwas montiert, miteinander verbunden wird. Dies dient der Erzeugung von Überraschungsmomenten und der Annäherung an die moderne, immer komplizierter werdende Wirklichkeit. ${ }^{25}$

Die Montage ist eine Ausprägung der Simultantechnik, d. h. Simultantechnik ist der Oberbegriff. Es werden Dinge oder Handlungen, die eigentlich bezüglich ihres Ortes, der Zeit oder der Kausalität auseinanderliegen, zusammengesetzt. Das Thema wird aus verschiedenen Perspektiven beleuchtet.

Der Wirt hatte zuletzt so leise mit ihr gesprochen, im Ton des geheimen Einverständnisses, daß sie unmöglich etwas von der Unterhaltung hatten verstehen können. Judith stocherte in der Omelette herum. Sie hörte die Stimme des Radioansagers: Wir bringen jetzt ein Lied aus dem Tonfilm >Heimat gesungen von Zarah Leander. (Sansibar, S. 74)

Gleich darauf setzte die Musik ein. Judith sah, daß die Schweden sich zum erstenmal gegenseitig anblickten. Dann sahen sie wieder geradeaus, mit Blicken, die nun nicht mehr hindämmerten, sondern mit starren, verlegenen und belästigten Blicken, als die Sängerin einsetzte: Mann nennt mich Miss Jane, die berühmte, bekannte, yes sir. (Sansibar, S. 74)

Hier wird durch Zeilen aus einem Lied unterbrochen, in dem es um eine Frau geht, die sich nicht um moralische Normen schert. Hier scheint die Mischung von verschiedenen künstlerischen Verfahren vorzuliegen, um die perspektive zu verdeutlichen.

Traditionelle Elemente werden durch den Einsatz von Symbolen konkretisiert. Hinsichtlich des Symboleinsatzes ist Andersch aber eher ein traditioneller als moderner Erzähler.

Die geografische Gegebenheiten wie Landschaften, Städte, Flüsse und bestimmte Gebäude spielen in Anderschs Roman eine sehr bedeutende Rolle. Sie sind nicht nur der Raum, in dem das Geschehen stattfindet, sondern Verkörperung von bestimmten Gefühle oder Wunschvorstellungen manchmal auch von Ideen.

Rerik ist ein Ort der Enge, Abhängigkeit und Bedrohung

Da man jedoch bedroht war, dachte Gregor, war nichts etwas anderes. Die Gegenstände schlossen sich in die Namen, die sie trugen, vollkommen ein. Sie wiesen nicht über sich selbst hinaus[...] Ein Haus würde ein Haus sein. (Sansibar, S. 8) 
Aber Rerik ist auch die Möglichkeit zur Freiheit, wo alle Figuren ihre Schritte zur Flucht beginnen.

Unter der Bedrohung sieht man die Dinge sehr intensiv, wie sie wirklich sind. Für Pastor Helander ist ein Haus eben nicht ein Haus, sondern die Wand seines Gotteshauses ist ihm sogar eine Tafel, auf der er die Handschrift Gottes erwartet. Judiths Mutter hat die Türme Reriks als wunderbare rote Monster bezeichnet, die man streicheln kann. Die Mutter war damals nicht bedroht, aber Judith sieht diese Türme wie ein böse Monster, das eine Stadt getötet hat. Sie unterdrücken die Freiheit; sie beherrschen und kontrollieren die Leute und die See vor der Stadt. Sie lassen keine Träume von politischer Freiheit zu. Sie sind hilfreich als Seezeichen, als Teil der Kirche Helanders und als Heimat des Klosterschülers; als Platz der geistigen Freiheit.

Die offene Ostsee ist blau und eisig. Das ist ein Ort des Todes, aber auch Wendepunkt der Freiheit für alle Personen „Ostsee, die eine dunkle Wand ohne Tür war" (Sansibar, S. 82). Für Gregor ist Trasovka wichtig, weil dort sein Verrat begonnen hatte, was sich in Rerik vollenden sollte. Sie ist das Leitbild seines Widerstandes gegen die Partei. Tarasovka ist ein Symbol der Freiheit und Lösung von kommunistischen Parteigrenzen „Tarasovka, weil dort sein Verrat begonnen hatte. “ (Sansibar, S. 23). Der goldene Schild symbolisiert durch eine Farbe den Verrat ind die Hoffnung auf Freiheit.

Sansibar ist ein Ort der Freiheit. Der Junge will fort von Rerik, weil er von Sansibar gehört hatte, Sansibar hinter der offenen See. Mississippi und Sansibar sind Bezeichnung für eine gedachte Gegenwelt zu Rerik. Rerik ist für den Junge langweilig und bedeutet auch Abhängigkeit von der Mutter und Knudsen, aber Sansibar steht für Abenteuer. Aber er erkennt, dass die Welt der Abenteuerbücher wirklich beendet ist. Der Titel ,Sansibar oder der letzte Grund“ bezieht sich auf einen Tagtraum des Jungen, in dem Sansibar jedoch weniger ein konkretes Ziel ist als vielmehr der utopische Ort einer besseren Zukunft.

\section{Fazit:}

Geistesgeschichtliche Voraussetzungen für den modernen Roman:

- Verlust einer geschlossenen Gesellschaft.

- Verlust eines einheitlichen und umfassenden Glaubens- und Weltverständnisses.

- Individualisierung und Depotenzierung des Helden (Antiheld).

- Erschwerung bzw. Unmöglichkeit der Auffindung des Lebenssinns.

- Skepsis gegenüber der Welt bzw. Ablehnung der Welt.

- Lebenssinn im antiken und mittelalterlichen Epos bekannt und erreichbar; im traditionellen Roman nicht von vornherein feststehend, aber erkennbar und aufzufinden; im modernen Roman Möglichkeit der Sinnfindung negiert bzw. überhaupt nicht diskutiert. 


\section{Der moderne Roman ist vor allem durch folgende Wesenszüge gekennzeichnet:}

\section{Abschied vom Helden}

Statt des sich in allen Lebenslagen und Konflikten behauptenden großen Individuums, des im Positiven wie im Negativen überragenden Menschen kennt der moderne Roman als „Helden“ nur den Durchschnittsmenschen mit allen Schwächen und Gebrechlichkeiten: geängstigt, zerrissen, mittelmäßig, oft verzagend $\Rightarrow$ kein Identifikationsangebot.

\section{Abschied von der Fabel}

Statt des chronologisch und kausal angelegten Handlungsgefüges des traditionellen Romans überwiegt im modernen Roman das Unverbundene, Zufällige, Sprunghafte. Nicht ein kausal geordnetes Geschehen steht im Mittelpunkt, sondern das im Bewusstsein der Figuren sich spiegelnde Geschehen.

\section{Verkomplizierung des Erzählens}

Der moderne Roman wird weithin nicht chronologisch erzählt. Durch Rückblenden, Erinnerungsmonologe, Assoziationen etc. wird Vergangenes oft in den Fortgang der Handlung eingebaut.

\section{Literaturverzeichnis}

\section{Primärliteratur}

- Andersch, Alfred : Sansibar oder der letzte Grund. Zürich, Diogenes Verlag, 1970.

\section{Sekundärliteratur}

- Alo Allkemper, Norbert Otto Eke: Literaturwissenschaft, 3.Auflage, Wilhelm Fink GmH\&co. verlages. KG, 2004.

- Geist, Alexander: Sansibar oder der letzte Grund Alfred AnderschInhalt-Hintergrund-Interpretation, mentor Verlag GmbH, München, 2005.

- Klotz, Volker: Zitat und Montage in neuerer Literatur und Kunst. In: Literatur und bildende Kunst: ein Handbuch zur Theorie und Praxis eines komparatistischen Grenzgebietes. Herausgegeben von Ulrich Weisstein. Berlin: Erich Schmidt, 1992.

- Müller, Fred: Alfred Andersch Sansibar oder der letzte Grund:

\section{Lexika} Interpretation von Fred Müller,1.Auflag, München: Oldenbourg, 1988.

- Duden Deutsches Universalwörterbuch, 5. Aufl., Dudenverlag, Mannheim, Leibzig, Wien, Zürich, 2003.

- Wahrig, Gerhard: Wahrig Wörterbuch der deutschen Sprache. Deutscher Taschenbuch München Verlag GmbH\& Co.KG., 1978.

- Meyers Großes Taschenlexikon, lexikonverlag, 2005.

\section{Internetwebseiten}

- http://oregonstate.edu/instruct/ger343/roman.htm 
- http://www.kikuma.de/mainnavigation/aha/was-ist-ein-roman/

- http://www.pausenhof.de/referat/deutsch/der-roman/14419

- http://www.magicpoint.net/fingerzeig/literaturgattungen/roman/roman.htm

- http://www.buecherwiki.de/index.php/BuecherWiki/GeschichteDesRomans

- http://www.gutefrage.net/frage/was-ist-ein-roman

- http://www.buecherwiki.de/index.php/BuecherWiki/AufbauDesRomans

- http://www.kkgwhv-schule.kwe.de/deutsch/refmdr.htm

- http://www.magic-point.net/fingerzeig/literaturgattungen/roman/roman theorie/roman-theorie.html

- http://www.magicpoint.net/fingerzeig/literaturgattungen/roman/roman_erge bn1/roman_ergebn1.html

- http://www.schreiben10.com/referate/Deutsch/4/Alfred-Andersch--Sansibar-oder-der-letzte- Grund-reon.php

- http://www.litde.com/jugendlexikon-literatur/simultantechnik.php

- http://de.wikipedia.org/wiki/Montage-\%28Literatur\%29 
1 -http://oregonstate.edu/instruct/ger343/roman.htm 29/7/2012.

2 -Vgl. Spörl, Uwe: Basislexikon literaturwissenschaft, 2.durchgesehene Auflage, Verlag Ferdinand Schöningh, Paferborn, 2006, S. 303.

3 -http://www.kikuma.de/mainnavigation/aha/was-ist-ein-roman/ 03/08/2012.

4 -http://www.pausenhof.de/referat/deutsch/der-roman/14419 05/08/2012.

5 -http://oregonstate.edu/instruct/ger343/roman.htm05/08/2012.

6 -http://www.magicpoint.net/fingerzeig/literaturgattungen/roman/roman.htm 06/08/2012.

7 -Vgl. Alo Allkemper, Norbert Otto Eke: Literaturwissenschaft, 3.Auflage, Wilhelm Fink GmH\&co. verlages. KG, 2004, S. 111.

8 -Vgl. Meyers Großes Taschenlexikon, lexikonverlag, 2005, S. 6390.

9-http://www.buecherwiki.de/index.php/BuecherWiki/GeschichteDesRomans08/08/2012.

10 -http://www.gutefrage.net/frage/was-ist-ein-roman10/08/2012.

11-http://www.buecherwiki.de/index.php/BuecherWiki/AufbauDesRomans10/08/2012

12 -http://www.kkgwhv-schule.kwe.de/deutsch/refmdr.htm 21/9/2012.

13-http://www.magic-point.net/fingerzeig/literaturgattungen/roman/roman theorie/romantheorie.html12/12/2012.

14 -Ebd.

15 -http://www.kkgwhv-schule.kwe.de/deutsch/refmdr.htm 2/3/2013.

16-Vgl.Geist, Alexander: Sansibar oder der letzte Grund Alfred Andersch- InhaltHintergrund-Interpretation,mentor Verlag GmbH, München, 2005, S. 28.

17-http://www.magicpoint.net/fingerzeig/literaturgattungen/roman/roman_ergebn1/ roman_ergebn1.html 5/3/2013.

18- http://www.schreiben10.com/referate/Deutsch/4/Alfred-Andersch---Sansibar-oder-derletzte- Grund-reon.php 5/3/2013.

19 -Vgl. Müller, Fred: Alfred Andersch Sansibar oder der letzte Grund: Interpretation von Fred Müller,1.Auflag, München: Oldenbourg, 1988, S. 47.

20 -Andersch, Alfred: Sansibar oder der letzte Grund, Roman,Diogenes Verlag AG Zürich, 1970, S. 17. [Im folgenden mit der Sigle Sansibar+ Seitenzahl zitiert]

21 -http://www.litde.com/jugendlexikon-literatur/simultantechnik.php 2/4/2013

22 -Meyers Großes Taschenlexikon in 24 Bänden. Herasugegeben von Rudolf Ohlig, 5. überarbeitete Auflage, Mannheim 1995, S. 25.

23 -Ebd.

24 -Klotz, Volker: Zitat und Montage in neuerer Literatur und Kunst. In: Literatur und bildende Kunst: ein Handbuch zur Theorie und Praxis eines komparatistischen Grenzgebietes. Herausgegeben von Ulrich Weisstein. Berlin: Erich Schmidt 1992, S. 180.

25 -http://de.wikipedia.org/wiki/Montage-\%28Literatur\%29 2/4/2013. 